\title{
Mass Spectrometric Study of Photoionization. X. Hydrogen Chloride and Methyl Halides
}

\author{
Morris Krauss, James A. Walker, and Vernon H. Dibeler \\ Institute for Basic Standards, National Bureau of Standards, Washington, D.C. 20234
}

(March 12, 1968)

\begin{abstract}
Photoionization efficiency curves are obtained for hydrogen chloride and several methyl halides, both ordinary and deuterated, from ionization threshold to $600 \AA$. Discussion is given on electronic structure of ions, autoionizing Rydberg states, and line-shape behavior. Ionization energies, heats of formation of ions, and bond dissociation energies are tabulated without regard to distribution of energy in internal modes, in relative kinetic energy, or other possible modes.
\end{abstract}

Key Words: Dissociation energies; HCl; ionization; mass spectrometry; methyl halides; vacuum ultraviolet spectroscopy.

\section{Introduction}

The purpose of this paper is to analyze the photoionization efficiency (PE) curves of $\mathrm{HCl}, \mathrm{CH}_{3} \mathrm{~F}$, $\mathrm{CD}_{3} \mathrm{Cl}, \mathrm{CH}_{3} \mathrm{Cl}, \mathrm{CH}_{2} \mathrm{DCl}, \mathrm{CHD}_{2} \mathrm{Cl}$, and $\mathrm{CH}_{3} \mathrm{Br}$ measured from their thresholds to $600 \AA$. Analysis of such a series is usually undertaken in the expectation of the similarities that are revealed. Such a motive is clearly revealed in early studies of the Rydberg series in the hydrogen [1] ${ }^{1}$ and methyl [2] halides. Understanding of the electronic structure of these molecules is clearly aided by studying trends in the series. Up to a point the same philosophy will hold for photoionization studies.

However, the ion production channels are not always dominant and the regularities in electronic behavior are not always mirrored in the PE curves. In particular, the ion-pair production yields very unusual qualitative behavior. The structure of the ion-pair PE curve [3] for $\mathrm{CH}_{3} \mathrm{Cl}$ is so rich that further investigation into this process was instituted by examining the deuterated methyl chloride molecules and by searching for ionpair processes in the fluoride and bromide. Methyl iodide has previously been studied [4]. In order to complete the investigation, all ions from the methyl halides were studied and $\mathrm{HCl}$ was also examined to assist the interpretation of the $\mathrm{CH}_{3} \mathrm{Cl}$ spectra. The $\mathrm{HCl} \mathrm{PE}$ curves were found to possess a rich autoionization structure interesting in itself.

At present, experimental resolution is insufficient to warrant quantitative spectroscopic analysis. However, sufficient results are available to warrant a comprehensive qualitative analysis of hydrogen chloride and the methyl halides. The analysis will report and speculate on the following:

1. Electronic structure of the ions and autoionizing states.

2. Bond energies and ionization thresholds.

3. Line-shape behavior especially in neighborhood of the ${ }^{2} \Sigma^{+} \mathrm{HCl}$ threshold.

\section{Experimental Procedure}

Photoionization efficiency curves were obtained by means of the combined vacuum ultraviolet monochromator and mass spectrometer described in detail in the first paper of this series [5], but including minor modifications reported in more recent studies [6]. Data were obtained with an optical resolution of about $1 \AA$, with an uncertainty in the absolute wavelength of $1 \AA$, or less over all wavelengths. In the absence of systematic error, the uncertainty in the reported relative ionization efficiency (ions per photon transmitted through the ion source) is of the order of 3 percent. It is ascribed principally to the uncertainty in measuring the numbers of electrons photoejected from a tungsten photon detector.

The hydrogen chloride and the ordinary methyl halides were reagent gases ${ }^{2}$ obtained from the Matheson Company. The minimum purity as stated by the supplier was generally better than 99 percent. Mass

\footnotetext{
Figures in brackets indicate the literature references at the end of this paper.

${ }^{2}$ Certain commercial materials are identified in this paper in order to adequately specify the experimental procedure. In no case does such identification imply recommendation or endorsement by the National Bureau of Standards, nor does it imply that the material identified is necessarily the best available for the purpose.
} 
spectrometric analysis showed no significant amounts of interfering impurities. The deuteromethyl halides were obtained from Merck, Sharp, and Dohme, Ltd. Isotopic purities were stated to be better than 98 atom percent $\mathrm{D}$, and these were confirmed by mass spectra. Chemical purities were apparently comparable.

The following conversion factors are used in this report:

$1 \mathrm{eV}$ molecule ${ }^{-1}=96.4870 \mathrm{kJmol}^{-1}, 1 \mathrm{kcal} \mathrm{mol}^{-1}=$

\section{$4.1840 \mathrm{kJmol}^{-1}$}

\section{3. $\mathrm{HCl}$}

Electronic Structure and PE Curves. Mulliken [7] first described the electronic structure of $\mathrm{HCl}$ in terms of molecular orbitals (MO). He noted that one-electron ionization would yield two ion states that are in the energy range of the present investigation. The electronic structure is given as

$$
\begin{array}{cccc}
\text { KL } & 4 \sigma^{2} & 5 \sigma^{2} & \pi^{4} \\
& 30.39 & 16.26 & 12.70
\end{array}
$$

where the ionization energies in $\mathrm{eV}$ (theoretical estimate for $4 \sigma[8]$ ) are given below the orbital symbol. Transitions between the ${ }^{2} \Sigma^{+}$and ${ }^{2} \Pi$ levels of the ion states were analyzed by Norling [9] and the asymptotic behavior and spectroscopic constants given [10]. The inner ionization energy was thus determined quite accurately relative to the ion ground-state energy and the doublet splitting, ${ }^{2} \Pi_{3 / 2}-{ }^{2} \Pi_{1 / 2}$, was also determined.

Although a Rydberg series has not been analyzed for HCl, Price [1] deduced a value of $12.84 \mathrm{eV}$ for

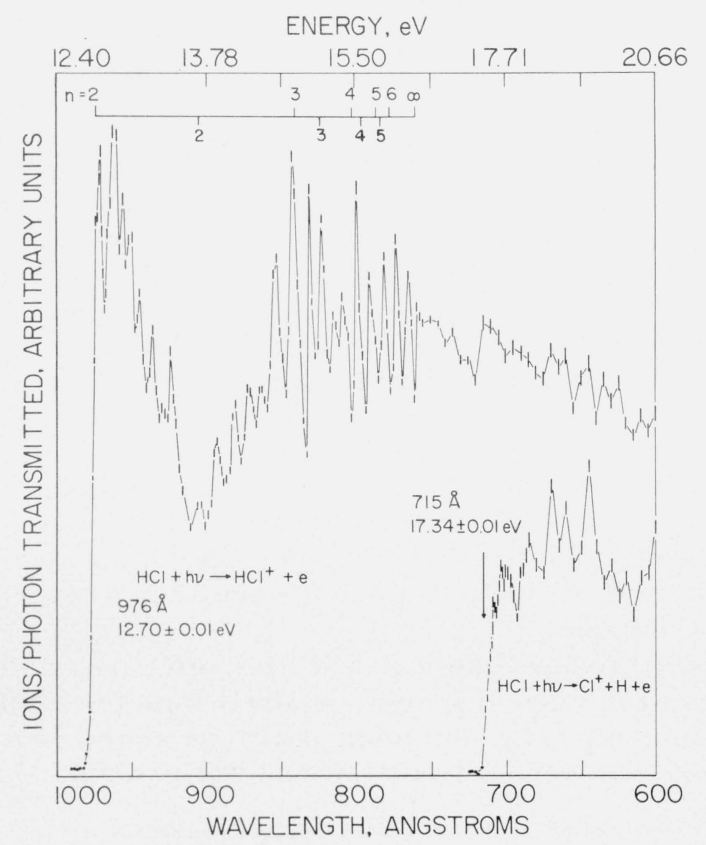

Figure 1. Photoionization yield curves for the $\mathrm{HCl}^{+}$and $\mathrm{Cl}^{+}$ions of hydrogen chloride. the ionization onset. Electron impact values have been somewhat lower. Morrison [11] determined values of $12.53 \pm 0.09 \mathrm{eV}$ and $12.78 \pm 0.03 \mathrm{eV}$ using the linear extrapolation and critical slope methods, respectively; whereas Fox [12] reports $12.56 \pm 0.1$ $\mathrm{eV}$. Considerable curvature in the threshold ionization curve was attributed by Fox to the doublet electronic structure. He then estimated the doublet splitting to be $0.3 \mathrm{eV}$ which is much larger than the value of $0.08 \mathrm{eV}$ determined spectroscopically. There is also disagreement between the spectroscopic and electron impact values for the energy of the first excited state of $\mathrm{HCl}^{+}$. Fox and Morrison find indications of a second ionization limit $1.5 \mathrm{eV}$ above threshold and Norling reports a value of $3.56 \mathrm{eV}$ for the interval. These discrepancies are reminiscent of similar problems that have been traced to autoionization contributions and this is apparently the case here. As we shall see below, autoionization is the dominant feature of the photoionization efficiency curve below $20 \mathrm{eV}$.

As rotational transitions are not resolved and vibrational bands are probably superimposed, the assignment of transitions is, at best, speculative. Intensities are not a sure guide to selecting a given vibrational band accompanying Rydberg excitation. However, we can use the known ionization energy for the ${ }^{2} \Sigma^{+}$state to estimate quantum defects and Rydberg energies. Selected wavelengths of the peak maxima are given in table 1. As can be seen in figure 1, several series of peaks seem to be connected and are probably vibrational progressions accompanying either one Rydberg transition or several closely spaced ones. We particularly have in mind the progressions beginning at $971 \AA$, the one at $904 \AA$, and finally the one at $854 \AA$. Assuming that the peaks at 904 and 971 represent the $0-0$ vibrational bands to electronic states to two separate Rydberg series, we can use the known ionization energy to fit quantum defects. A list of the estimated energies of the other members in

TABLE 1A. Selected wavelengths of peak maxima in $\mathrm{HCl}^{\text {a }}$

\begin{tabular}{|c|c|c|c|c|c|}
\hline $\begin{array}{c}\text { Peak } \\
\text { wavelength } \\
(\AA)\end{array}$ & $\begin{array}{c}\text { Peak } \\
\text { separation } \\
(\mathrm{eV})\end{array}$ & $\begin{array}{l}\text { Effective } \\
\text { quantum } \\
\text { number }\end{array}$ & $\begin{array}{c}\text { Peak } \\
\text { wavelength } \\
(\AA)\end{array}$ & $\begin{array}{c}\text { Peak } \\
\text { separation } \\
(\mathrm{eV})\end{array}$ & $\begin{array}{l}\text { Effective } \\
\text { quantum } \\
\text { number }\end{array}$ \\
\hline $\begin{array}{l}971.5 \\
962 \\
956 \\
952.5 \\
945 \\
936 \\
932 \\
925\end{array}$ & $\begin{array}{c}0.12 \\
.08_{1} \\
.04_{8} \\
.10 \\
.13 \\
.05_{6} \\
.10\end{array}$ & $\sim 1.9$ & $\begin{array}{l}904 \\
893 \\
882 \\
872 \\
863 \\
854 \\
842 \\
831 \\
823\end{array}$ & $\begin{array}{r}0.17 \\
.17 \\
.15 \\
.15 \\
.15 \\
.21 \\
.19 \\
.15\end{array}$ & $\sim 2.3$ \\
\hline
\end{tabular}

a Peak wavelengths are estimated from figure 1 for apparent vibrational series originating at $971.5,904$, and $854 \AA$. The effective quantum number at the origin is estimated from the known ${ }^{2} \Sigma^{+}$ionization potential.

TABLE 1B. Estimate of higher Rydberg levels in $\mathrm{HCl}^{\text {a }}$

\begin{tabular}{c|c||c|c}
\hline \hline $971 \AA$ Series $n^{*}$ & Rydberg wavelength $(\AA)$ & $904 \AA$ Series $n^{*}$ & Rydberg wavelength $(\AA)$ \\
\hline & 971 & & \\
1.94 & 841 & 2.28 & 904 \\
2.94 & 803 & 3.28 & 824 \\
3.94 & 787 & 4.28 & 796 \\
4.94 & 5.28 & 783 \\
\hline
\end{tabular}

a Estimate of band origins for two Rydberg series with constant quantum defects of 0.06 and 0.72 respectively. 
table $1 \mathrm{~B}$ shows how difficult it is to separate and to assign states in the $815 \AA$ region and beyond. Primarily, the difficulty is due to the large number of vibrational states that are excited.

Franck-Condon factors have been reported [13] for the transitions to the ${ }^{2} \Sigma^{+}$ion and we will assume that the Rydberg behavior is similar. The ${ }^{2} \Sigma^{+} \omega_{e}$ is about $1605 \mathrm{~cm}^{-1}$ with a fairly large $\omega_{e} x_{e}$ of $40 \mathrm{~cm}^{-1}$. The levels beginning at $904 \AA$ are spaced at about $0.15 \mathrm{eV}$ and those beginning at $854 \AA$ average a little larger, closer to $0.20 \mathrm{eV}$. There seems little doubt that these two states are Rydbergs with the ${ }^{2} \Sigma^{+}$ion as the limit. The $904 \AA$ progression may be the basis of the second break in the electron-impact ionization efficiency curve found by Fox and by Morrison.

Speculative assignments can be made now by considering Rydberg orbitals in the field of the ${ }^{2} \Sigma^{+}$core and the molecular states that correlate with appropriate asymptotic atom states. In the molecular orbital scheme the ${ }^{2} \Sigma^{+}$ion state arises from a single-electron ionization from the bonding $5 \sigma$ orbital. This state correlates asymptotically with the $\mathrm{H}^{+}+\mathrm{Cl}\left({ }^{2} \mathrm{P}\right)$ atoms rather than the $\mathrm{H}\left({ }^{2} \mathrm{~S}\right)+C l^{+}\left({ }^{3} \mathrm{P}\right)$ as does the ${ }^{2} \Pi$ ion ground state. Superficially one would expect that the ${ }^{2} \Sigma+$ Rydberg states would correlate with the atomic hydrogen Rydberg states and ground state atomic chlorine. The noncrossing rule technically forbids this but near the molecular equilibrium position a catalog of possible molecular states can be made from the attachment of atom-like Rydberg orbitals with considerable hydrogen character that correlate to the asymptotic hydrogen Rydbergs. Since the ground state electronic distribution is well represented by the ionic formula $\mathrm{H}^{+} \mathrm{Cl}^{-}$, excitation of hydrogenic Rydbergs results in large transition moments of the charge transfer type.

The $971 \AA$ system is found to have an effective quantum number less than 2. Autoionization between the ${ }^{2} \Pi_{1 / 2}$ and ${ }^{2} \Pi_{3 / 2}$ doublet analogous to that in the rare gas atom is probably not observed because of the disparity that will be shown in the magnitude of the cross sections to the direct ${ }^{2} \Pi$ and ${ }^{2} \Sigma+$ ionization continua. Curvature found by Fox is probably unrelated to the doublet splitting. It is due more likely to the autoionization peaks at threshold. Using peak maxima positions as the band origins, the energy separations in the $971 \AA$ system are not found to fit any regular pattern. Peaks are evidently not resolved and may be due to more than one electronic state. The $n p \sigma^{*}$-series first member would locate at this energy for a primarily $n=2$ hydrogen Rydberg. The MO designation is KL $4 \sigma^{2} 5 \sigma \pi^{4} 6 \sigma$ and overall symmetry is ${ }^{1} \Sigma^{+}$.

Table 2 shows the correlation rules and energies of the lowest asymptotic atom states. The ion-pair state yields a ${ }^{1} \Sigma^{+}$state but this correlates ultimately with the $\mathrm{V}^{1} \Sigma^{+}$state some $5 \mathrm{eV}$ lower in energy. This $\mathrm{V}$ state is the first Rydberg state (which will probably not fit the series well) for a series KL $4 \sigma^{2} 5 \sigma^{2} 2 \pi^{3} n \pi$. It has been connected to the $4 \sigma^{2} 5 \sigma 2 \pi^{4} 6 \sigma$ state [14] but we prefer to assign this MO configuration to the Rydberg observed at $971 \AA$. Note that there will be considerable configuration mixing at equilibrium
TABLE 2. Correlation of asymptotic atom states for $\mathrm{HCl}^{\text {a }}$

\begin{tabular}{|c|c|c|c|}
\hline & om states ${ }^{b}$ & Molecular correlation & Energy $(\mathrm{eV})$ \\
\hline $\begin{array}{l}\mathrm{Cl},{ }^{2} \mathrm{P}^{\circ} \\
\mathrm{H}=2\left\{^{{ }^{2} \mathrm{~S}}\right. \\
{ }_{2 \mathrm{P}^{\circ}}\end{array}$ & $\begin{array}{l}4 s\left({ }^{1} \mathrm{D}\right)^{2} \mathrm{D} \\
4 p\left({ }^{3} \mathrm{P}\right)^{2} \mathrm{D}^{\circ} \\
4 p\left({ }^{3} \mathrm{P}\right)^{2} \mathrm{~S}^{\circ} \\
4 p\left({ }^{3} \mathrm{P}\right)^{2} \mathrm{P}^{\circ} \\
4 p\left({ }^{1} \mathrm{D}\right)^{2} \mathrm{P}^{\circ} \\
4 p\left({ }^{1} \mathrm{D}\right)^{2} \mathrm{~F}^{\circ} \\
5 p\left({ }^{3} \mathrm{P}\right)^{2} \mathrm{D}^{\circ} \\
\cdot . \\
4 d\left({ }^{3} \mathrm{P}\right)^{2} \mathrm{~F}\end{array}$ & 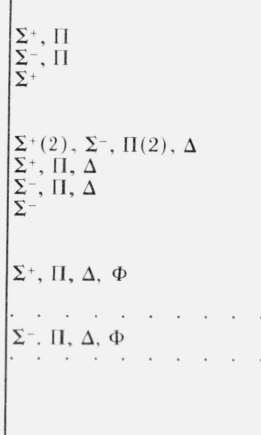 & $\begin{array}{r}0 \\
9.20 \\
9.98 \\
10.20 \\
\\
\\
10.43 \\
10.49 \\
10.57 \\
10.59 \\
11.69 \\
11.79 \\
11.83 \\
\quad \cdot . \\
12.00 \\
\\
12.09\end{array}$ \\
\hline
\end{tabular}

a Asymptotic excited atom states are combined with either ground state hydrogen or chlorine atoms except for the $\mathrm{H}^{+}+\mathrm{Cl}^{-}$ion-pair state. The excited orbital is noted and the parent ion state is given in parentheses. Relative energies are given for the lowest multiplet level. Note that the dissociation energy of $\mathrm{HCl}$ is $D_{0}(\mathrm{HCl})=4.33 \mathrm{eV}$.

Correlation states are not repeated for similar pairs of atom states. A number of levels are deleted as the density of levels increases but the positions of the $\mathrm{Cl} 4 d$ and $\mathrm{H}(n=3)$ states are given.

positions as well as at large internuclear separations. If there is considerable ionic character to this state the dissociation energy would exceed that of the ${ }^{2} \Sigma+$ ion and the vibrational spacing would also be smaller than that of the ion. The lower (infinity of) ${ }^{1} \Sigma+$ Rydberg states go asymptotically to the chlorine and hydrogen Rydberg asymptotic states producing complicated interactions at large $\mathrm{R}$ distances. However, the $(5 \sigma 6 \sigma)_{s}$ configuration should dominate in the neighborhood of the first ionization threshold. Note that a direct calculation [14] of the oscillator strength yields about 1.5 for this transition and 0.005 for the $2 \pi \rightarrow 6 \sigma$ transition explaining the relative insignificance of the direct ${ }^{2} \Pi$ ionization and the $\Pi_{3 / 2}-\Pi_{1 / 2}$ autoionization.

Comparison of the effective quantum number in table $1 \mathrm{~A}$ with those for the Rydberg states of $\mathrm{H}_{2}$ are quite suggestive. Effective quantum numbers for the $n$ p $\sigma^{*}$ series in $\mathrm{H}_{2}$ are $1.79,2.80, \ldots$ and the values $2.08,3.08,4.08, \ldots$. fit the $n p \pi^{*}$ series. Evidently the 971 and $854 \AA$ progressions arise from the same Rydberg series but either the $2 p \sigma(v=0)$ bands must be below the ionization limit or the $v=0$ band is obscured for the $3 p \sigma$ progression in order that the quantum defect decrease as a function of increasing $n$. The structure of HCl Rydbergs does not seem to differ from that of $\mathrm{H}_{2}$. Two series, $n p \sigma$ and $n p \pi$, are suggested with the very approximate band origins given in table $1 B$.

The asymptotic dissociation fragments for these Rydbergs cannot easily be inferred. If the progression for the $2 p \pi$ state really begins at $904 \AA$, then the dissociation energy into $\mathrm{H}(n=2)$ is only $0.9 \mathrm{eV}$ which is inconsistent with the observed vibrational spacing. Fortunately, the asymptotic behavior of the electronic energy curve is of little importance in this experiment as long as the bands cannot be resolved.

The vibrational progression at $854 \AA$ can be associated with a quantum-number three state which would primarily consist of $3 p$ hydrogenic orbitals. If the peaks at $854,842,831$, and $823 \mathrm{~A}$ are attributed to vibra- 
tional bands of one electronic state then the vibrational spacings of about $0.21,0.19$, and $0.15 \mathrm{eV}$ are quite consistent with vibrational spacing for the ${ }^{2} \Sigma+$ ion. However, the density of resonance states is high in this region and assignments are difficult to make. The $854 \AA$ series apparently belongs to the same series as the one at threshold and the two smaller peaks just below $800 \AA$ could fit the series with the first member at $904 \AA$. Speculation on the remainder of the peaks before the ${ }^{2} \Sigma^{+}$threshold would be fruitless except to note that if only hydrogen Rydbergs play an important role then there would only be the two dominant $\mathrm{HCl}$ $\left({ }^{2} \Sigma^{+}\right)$Rydberg series. It is possible that Rydbergs which lead to a bound ion state or states which go asymptotically to the $\mathrm{Cl}^{+} 3 p^{4}{ }^{1} \mathrm{D}$ state may be present with small probability but sufficient to disturb the regularity of the vibrational progressions.

The poorly resolved peaks that begin at about $714 \AA$ are found in both the parent ion and the $\mathrm{Cl}^{+} \mathrm{PE}$ curves. The resonance states contribute to both channels and all the open channels appear to have resonances at the same energy. The resonance states are probably Rydberg states leading to bound-ion states that correlate with both the $\mathrm{Cl}^{+}\left(3 s^{2} 3 p^{4}{ }^{1} \mathrm{D}\right.$ and $\left.{ }^{1} \mathrm{~S}\right)$ atoms. Predissociation into the ion state may be enhanced by the ${ }^{2} \Sigma^{-}$state that arises from ground state $\mathrm{Cl}^{+}\left({ }^{3} \mathrm{P}\right)$.

The last feature of interest is the lack of structure just beyond the second ionization threshold. At least at this level of optical resolution there is a sudden break in the PE curve as the autoionizing peaks have apparently reached their limit. This is unusual since the Franck-Condon factors would lead one to expect structure beyond the ionization limit. Speculation on this feature is deferred to the section on autoionization.

\section{Line-Shape Behavior}

The theory of the configuration interaction of a single resonance with many continua has been given by Fano [15]. The basic result is that configuration interaction of a single discrete state with two or more continua is shown to be equivalent to interaction of a single discrete level with one continuum. This continuum is a linear combination of uncoupled standingwave continua weighted by the discrete state-continuum interaction. If coupling is considered, the results are somewhat more complicated but have been considered by Fano and Prats [16] and Mies [17]. The only parameter that will concern us here is $q$. This is defined as

$$
q=\frac{\left(\Phi|z| \Psi_{0}\right)}{\pi \Sigma_{j}\left(\phi|\mathrm{H}| \Psi_{j \mathrm{E}}\right)\left(\Psi_{j \mathrm{E}}|z| \Psi_{0}\right)}
$$

If we ignore the principal-part integral in (1) then the numerator is just the transition probability to the unperturbed embedded bound-Rydberg electronic state. To a good approximation this integral is the product of an electronic integral and a vibrational overlap integral.
As we can see from figure 1 , the autoionization is most apparent as absorption-like peaks. This is associated with a large $q$. Calculation indicates that the electronic transition probability to the ${ }^{2} \Sigma+$ Rydbergs is large and the Franck-Condon calculations suggest that at least five vibrational resonance states should contribute. The denominator is more complicated but it is likely that the integral $\left(\Psi_{\pi_{\mathrm{E}}}|z| \Psi_{0}\right)$ is small as the transition integral of the first Rydberg to this state has been found to be small in a virtual Hartree-Fock calculation [14]. At an energy lower than the ${ }^{2} \Sigma^{+}$ threshold we have only one ionization continuum although there are predissociation and fluorescence channels that contribute to the width.

When the ${ }^{2} \Sigma^{+}$channel is open, there are effectively two terms in the denominator of (1). The direct transition integral to the ${ }^{2} \Sigma^{+}$continuum is probably large but because the coupling is due to Born-Oppenheimer breakdown, is offset to some extent by reduction in coupling to the continua from the resonance state, a high vibrational state of a ${ }^{2} \Sigma+$ Rydberg. Nonetheless, we can speculate that the $q$ in a vibrational progression changes quite drastically on passing through the ${ }^{2} \Sigma^{+}$ threshold since the denominator suddenly increases while the numerator is effectively unchanged. A small $q$ is associated with structure that does not give the appearance of absorption peaks. In fact for an extremely small $q$ the structure in the neighborhood of the resonance appears as ? "window" in the absorption. This region of the spectrum should be reexamined with higher resolution to determine whether this speculation has some basis or the qualitative change in the PE curve is due either to experimental line width resolution problems or to an incorrect value for the ${ }^{2} \Sigma^{+}$threshold.

\section{Methyl Halides}

Electronic Structure and PE Curves. The molecular orbital (MO) structure of the lowest energy orbitals in the halides was represented by Mulliken [7] as follows:

$$
(\mathrm{CH} e)^{4}(\mathrm{CX} a)^{2}(X e)^{4}
$$

The CHe MO is very similar to the bonding $\mathrm{CH}$ orbital in $\mathrm{CH}_{4}$ and will have an ionization threshold slightly less than that for $\mathrm{CH}_{4}$; i.e., $<12.7 \mathrm{eV}$. The Xe orbital is considered essentially the $n p$ atomic orbital of the halogen atom. The ionization threshold of the $\mathrm{CX} a \mathrm{MO}$ is not so easily estimated. It is the bonding $\mathrm{CX}$ orbitals and the ionization threshold should be higher than the mean of the dominant valence orbitals that make up the MO.

Mulliken noted that in the case of the fluoride the $\mathrm{CHe}$ orbital has the lowest ionization energy; whereas, for the bromide it is the $X e$ orbital. Although he indicated that the chloride most likely behaves like the bromide and iodide, it was noted that this case is more ambiguous. Price [2a] analyzed the Rydberg states of $\mathrm{CH}_{3} \mathrm{Cl}$ and concluded that it was the $X e$ electron that was ionized. He also determined the doublet splitting in the degenerate ${ }^{2} \mathrm{E}$ ion to be $800 \mathrm{~cm}^{-1}$. 
The only mass-analyzed photoionization work on the methyl halides is a study of $\mathrm{CH}_{3} \mathrm{I}$ which exhibited autoionization peaks which could be correlated with the spectroscopically observed Rydbergs converging to the ${ }^{2} \mathrm{E}_{1 / 2}$ ion limit. A preliminary communication on the ion-pair process in $\mathrm{CH}_{3} \mathrm{Cl}[3]$ showed that configuration interaction was important in this process. Comparison of the $\mathrm{CH}_{3} \mathrm{Cl}$ mass-analyzed ionization threshold also agreed with previous photoionization data and was somewhat lower than electron impact results.

Inspection of the parent-ion PE curves of the methyl halides (figs. 2 to 6 ) shows the difficulty in deducing electronic structure directly from structure in the PE curve. Autoionization or configuration interaction is prominent in the fluoride, chloride, and bromide PE curves. However, in only one case, the ionpair curves for $\mathrm{CH}_{3} \mathrm{Cl}$, are lines sufficiently resolved to permit comparison with previous spectroscopic results $[2 \mathrm{a}]$. Dibeler and $\mathrm{W}$ alker have already noted that the structure in this case is due to at least two series with the $\mathrm{CH}_{3} \mathrm{Cl}^{+}$ground-state as a limit.

In order to assist in the discussion of the results it is helpful to have estimates of the inner ionization energies. Approximate Hartree-Fock calculations were obtained for $\mathrm{CH}_{3} \mathrm{~F}$ and $\mathrm{CH}_{3} \mathrm{Cl}$ for this purpose. A brief description of these calculations has already been given [18] but for completeness eigenvalues are given in table 3. These results will be used to assist a detailed analysis of all aspects of the PE curves excepting the chloride ion-pair process.

TABLE 3. Orbital and ionization energies for $\mathrm{CH}_{3} \mathrm{~F}$ and $\mathrm{CH}_{3} \mathrm{Cl}{ }^{\text {a }}$

\begin{tabular}{l|c|c|c}
\hline \hline \multirow{2}{*}{ Molecule } & Symmetry & Energy & Ionization energy \\
\hline \multirow{2}{*}{$\mathrm{CH}_{3} \mathrm{~F}$} & & & \\
& $2 e$ & 14.3 & 12.50 \\
& $5 a_{i}$ & 17.8 & \\
$\mathrm{CH}_{3} \mathrm{Cl}$ & $3 e$ & 18.8 & 11.28 \\
& $3 e$ & 11.7 & \\
& $2 e$ & 14.4 & \\
& & 16.8 & \\
\hline
\end{tabular}

a All energies are in $\mathrm{eV}$.

\section{6. $\mathrm{CH}_{3} \mathrm{~F}$}

The onset of the parent PE curve (fig. 2) resembles that of $\mathrm{CH}_{4}$ as the ionized orbitals are similar. However, there are apparently five steps at intervals of 7 to $4 \AA$. The Hartree-Fock calculation would indicate that this progression includes excitation of the symmetric $\mathrm{C}-\mathrm{F}$ stretch as well as $\mathrm{C}-\mathrm{H}$ modes since the orbital is bonding with respect to $\mathrm{C}-\mathrm{H}$ and antibonding with respect to $\mathrm{C}-\mathrm{F}$. The autoionization noted by Chupka [19] for $\mathrm{CH}_{4}$ is also found in $\mathrm{CH}_{3} \mathrm{~F}$. However, it is not clear if all the features are analogous. The highest peak in the neighborhood of $925 \AA$ is at an energy where $n=3$ Rydbergs to the $5 a_{1}^{-1}$ ion are expected. In $\mathrm{CH}_{4}$, the inner ionization energy is much too high so that all autoionization is due to Born-Oppenheimer break- down in high vibrational states of $1 t_{1}^{-1}$ Rydbergs or, in the case of $\mathrm{CH}_{3} \mathrm{~F}$, from $1 e^{-1}$ Rydbergs.

There is a striking indication of the dominance of configuration interaction at threshold and a caution on the attribution of peak-like structure to autoionization and of step-like structure to vibrational progressions in direct ionization. Examination of the $\mathrm{CH}_{3}{ }^{+}$ and $\mathrm{F}^{-}$ion-pair PE curves shows a remarkable resemblance to the parent PE curve. A common precursor resonant-state must be assumed to contribute significantly to both ionization and ion-pair production. The ion-pair curves extend over some $2 \mathrm{eV}$ and the resonance states must change in character over this range. In particular, we note clear evidence of configuration interaction between 875 to $900 \AA$ with the Rydberg states leading to inner ionization thresholds.

The ionization of the $5 a_{1}$ electron is predicted to br $3.5 \mathrm{eV}$ above the first onset. This orbital is primarily the $\mathrm{C}-\mathrm{F}$ bonding orbital and the onset of the $\mathrm{CH}_{3}^{+}$evidently occurs from this excited ion surface. The ion ground state is bonding with respect to $\mathrm{C}-\mathrm{F}$ and the probability of exciting vibrational levels directly into the $\mathrm{CH}_{3}^{+}+\mathrm{F}$ channel is very small. Evidently the autoionizing higher $5 a_{1}^{-1}$ Rydbergs also do not contribute appreciably.

\section{7. $\mathrm{CH}_{3} \mathrm{Cl}$}

Several steps are evident at threshold in the $\mathrm{CH}_{3} \mathrm{Cl}^{+}$ $\mathrm{PE}$ curves shown in figure 3 . The first step interval is $0.09 \mathrm{eV}$ which is of the order of the spectroscopically deduced doublet splitting. However, no obvious autoionization structure appears in this interval for either $\mathrm{CH}_{3} \mathrm{Cl}^{+}$or $\mathrm{CD}_{3} \mathrm{Cl}^{+}$curves (figs. 3 and 4 ). Of probable significance is the observation that in the photoionization of argon obtained with the same apparatus used in this research, the doublet is not observed due to a lack of resolution.

Excitation of a $\mathrm{CH}_{3}$ deformation mode accompanies the transitions to the lower Rydberg states [2a]. The structure observed below $1090 \AA$ has energy separations of the order of $0.07 \mathrm{eV}$ or about one-half that observed in the Rydbergs. Of course, the second step in $\mathrm{CH}_{3} \mathrm{Cl}$ beyond the initial doublet could correlate with the vibrational excitation observed by Price. This transition would occur for a smaller energy in $\mathrm{CD}_{3} \mathrm{Cl}$ and the steps are not resolved in $\mathrm{CD}_{3} \mathrm{Cl}$. The $3 e$ orbital is primarily occupied by the chlorine lone-pair electrons but there is significant $\mathrm{Cl}-\mathrm{H}$ antibonding and $\mathrm{C}-\mathrm{H}$ bonding character. Deformation of the ${ }^{2} \mathrm{E}$ Jahn-Teller state should be appreciable and excitation of all the $\mathrm{C}-\mathrm{H}$ modes is expected.

The Hartree-Fock threshold for $7 a_{1}^{-1}$ is $2.7 \mathrm{eV}$ above the first ionization energy. The $7 a_{1}$ orbital is primarily the $\mathrm{C}-\mathrm{Cl}$ bonding orbital and thus long vibrational progressions are expected in the Rydbergs and the ion state. The ionization threshold region for $7 a_{1}^{-1}$ will also be overlapped by the autoionizing Rydbergs with the $2 e^{-1}$ limit. Since the $2 e_{1}$ orbital is the $\mathrm{C}-\mathrm{H}$ bonding orbital, the Rydberg and ion transitions will be accompanied by long vibrational progressions and it is 
ENERGY, eV

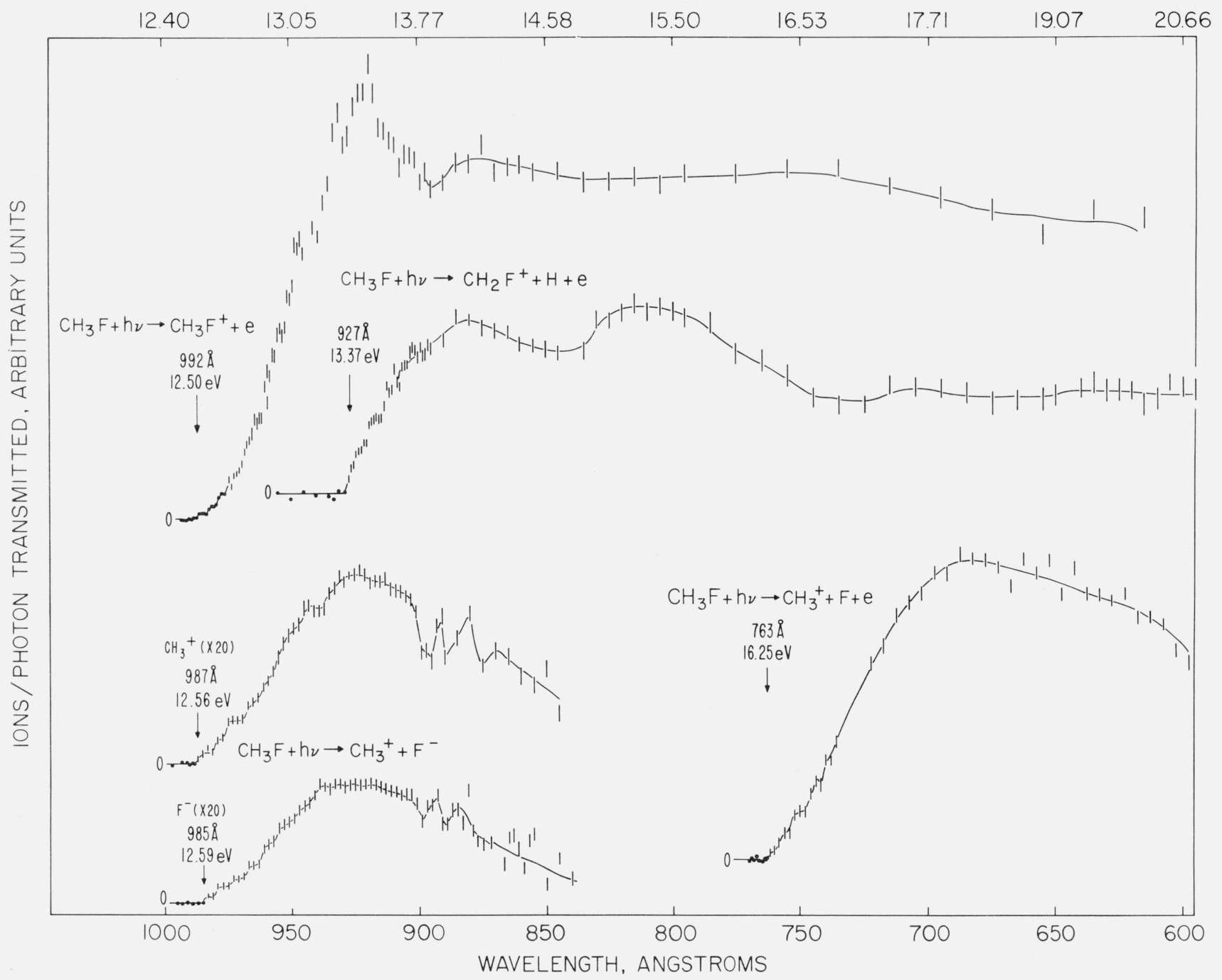

FIGURE 2. Photoionization yield curves for the molecule and some fragment ions of methyl fluoride. 
ENERGY, eV

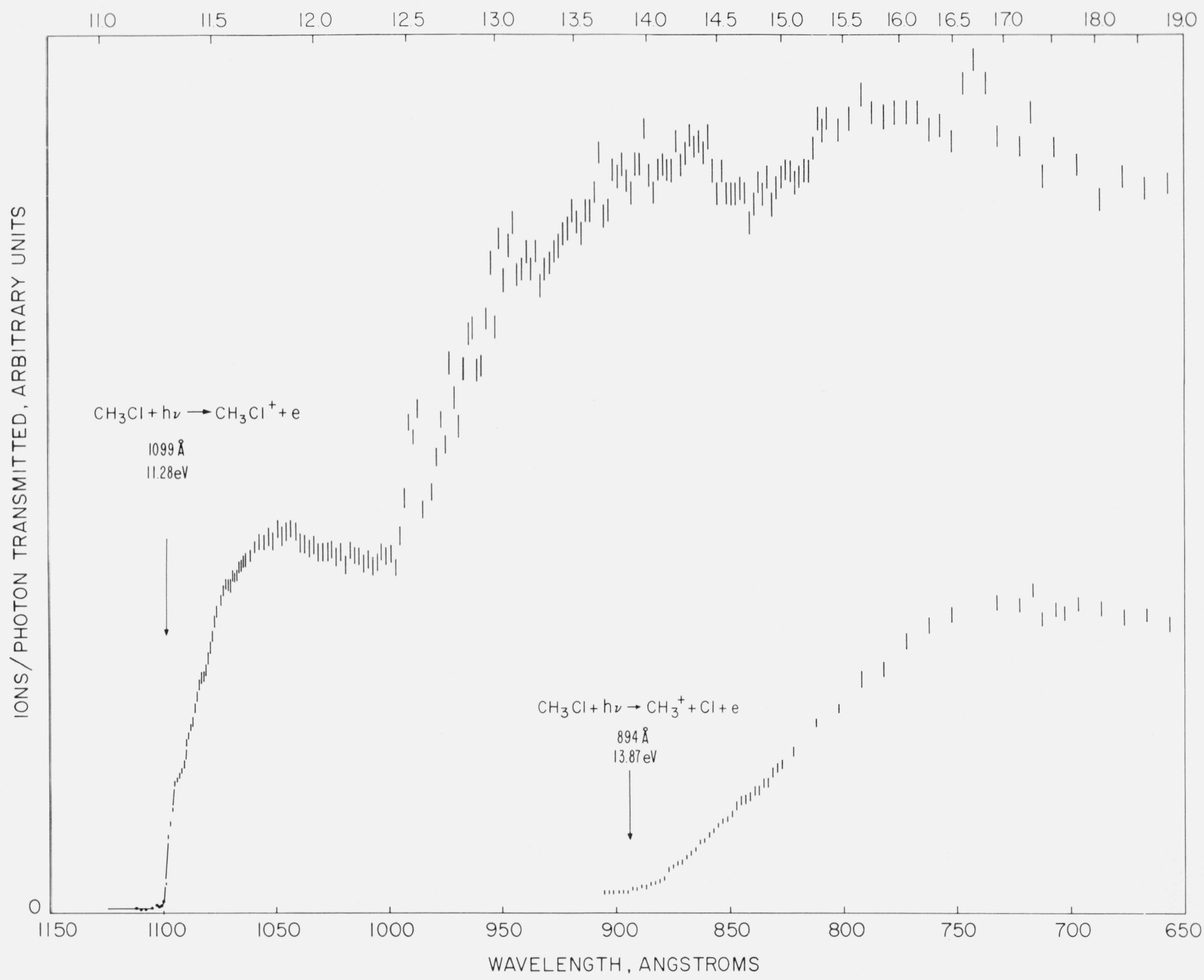

FIGURE 3. Photoionization yield curves of the $\mathrm{CH}_{3} \mathrm{Cl}^{+}$and the $\mathrm{CH}_{3}^{+}$ions of methyl chloride. 


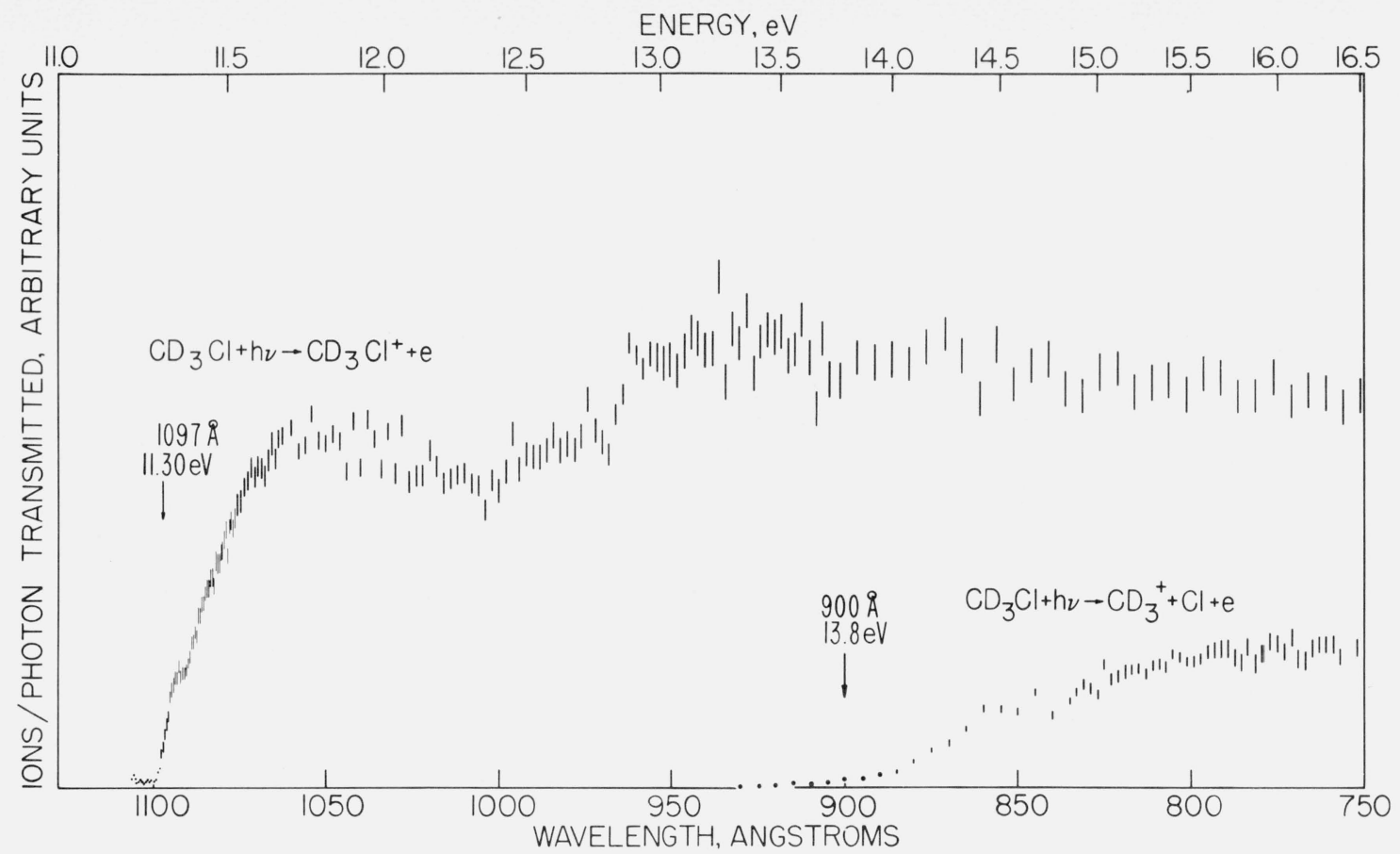

FIGURE 4. Photoionization yield curves for the molecule ion and the $\mathrm{CD}_{3}^{+}$ion of $\mathrm{CD}_{3} \mathrm{Cl}$.

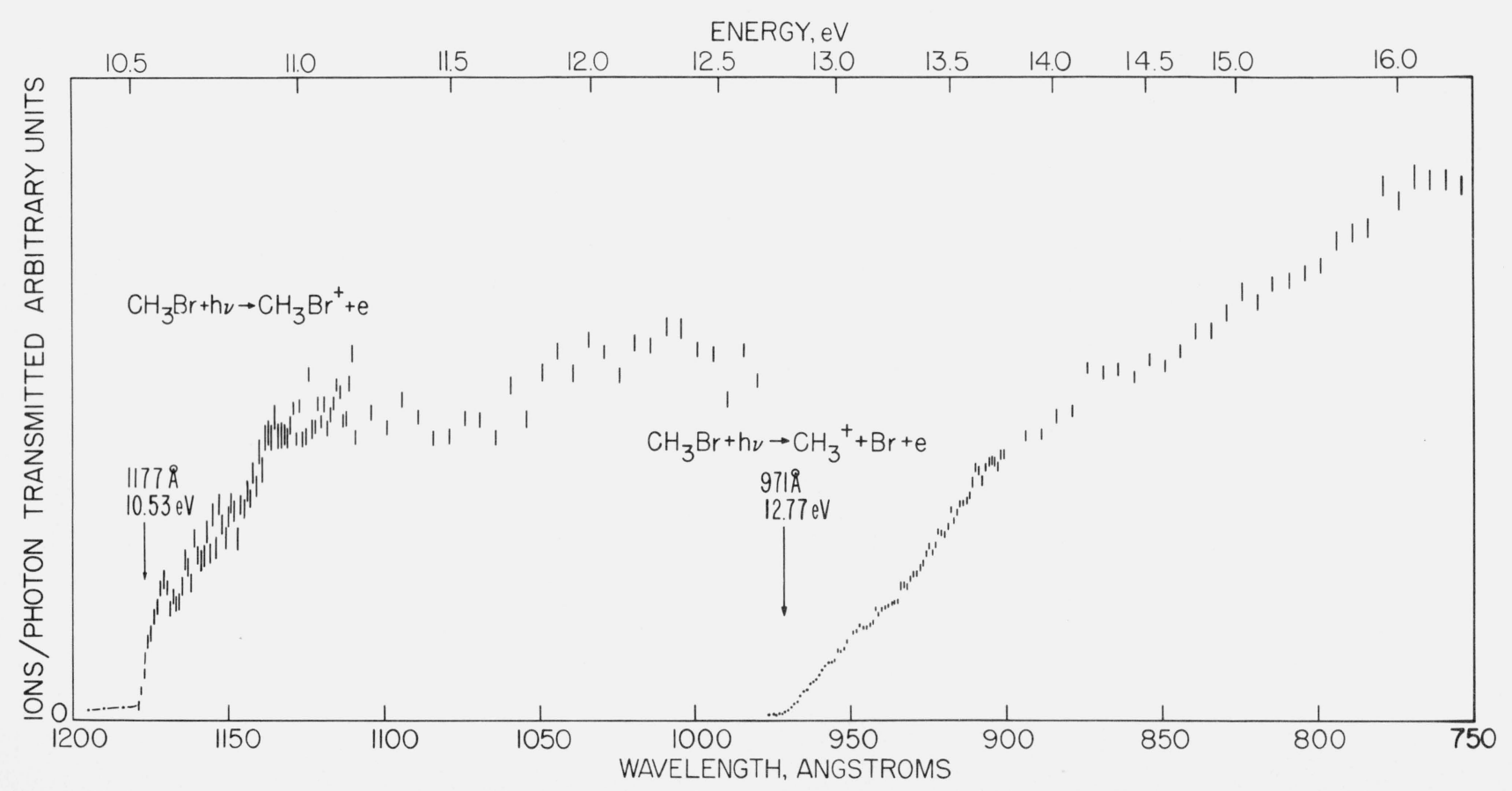

FIGURE 5. Photoionization yield curves for the molecule and methyl ions of methyl bromide.

not surprising that the ionization thresholds are obscured. It is also to be expected that the $\mathrm{CH}_{3}^{+}$ion is produced on the $7 a_{1}^{-1}$ surface but there is no evidence for the contributions of the autoionization of levels to this process.

\section{8. $\mathrm{CH}_{3} \mathrm{Br}$}

The methyl bromide results (fig. 5) confirm Price's conclusion that the nonbonding $\mathrm{Br}$ orbital is ionized and that no vibrational levels of the ion are excited. 
Contrary to the iodide or to photoionization measurements on $\mathrm{Kr}$, however, the Rydberg levels that lead up to $\Omega=1 / 2$ level do not appear as prominent absorptionlike autoionization levels. Whether this difference, too, is significant or an experimental artifact is not known.

The behavior of the PE curves for $\mathrm{CH}_{3} \mathrm{Br}^{+}$and $\mathrm{CH}_{3}^{+}$is very similar to that of $\mathrm{CH}_{3} \mathrm{Cl}$. The premise that autoionizing resonance states contribute to $\mathrm{CH}_{3}^{+}$formation is evidenced by the structure in the PE curve. The level spacing in the $\mathrm{CH}_{3}^{+} \mathrm{PE}$ curve would point to excitation of vibrational modes involving hydrogens.

\section{Ion-Pair Production in Methyl Chloride}

The ion-pair curves were verified by obtaining identical $\mathrm{PE}$ curves for the $\mathrm{CH}_{3}{ }^{+}$and the $\mathrm{Cl}^{-}$ions. In order to provide some insight into the mechanism, the ion-pair results of all deuterated species were obtained. The results for ordinary and deuterated com- pounds are shown in figures 6 and 7. There are several features of these curves that draw attention.

1. The general shape of the curves is a distorted Gaussian that is independent of isotope effect. This indicates a Franck-Condon transition from a ground vibrational polyatomic state to a continuum vibrational state in at least one degree of freedom for which the hydrogen atoms are not important.

2. The interference structure on the long wavelength side can be roughly correlated with the Rydbergs observed by Price. The peak values are compared in table 4. The structure disappears about $0.1 \mathrm{eV}$ beyond the first ionization threshold indicating that either excited Rydberg vibrational bands or the doublet splittings are being excited.

3. There is a pronounced isotope shift on the total ion-pair current. The ratio for $\mathrm{CH}_{3}: \mathrm{CH}_{2} \mathrm{D}: \mathrm{CHD}_{2}$ is $1.0: 0.9: 0.6$ as measured at the peak of the Gaussian. The $\mathrm{CD}_{3}^{+}$peak is observed but cannot be quantitatively measured because of the signal-to-noise limitation.

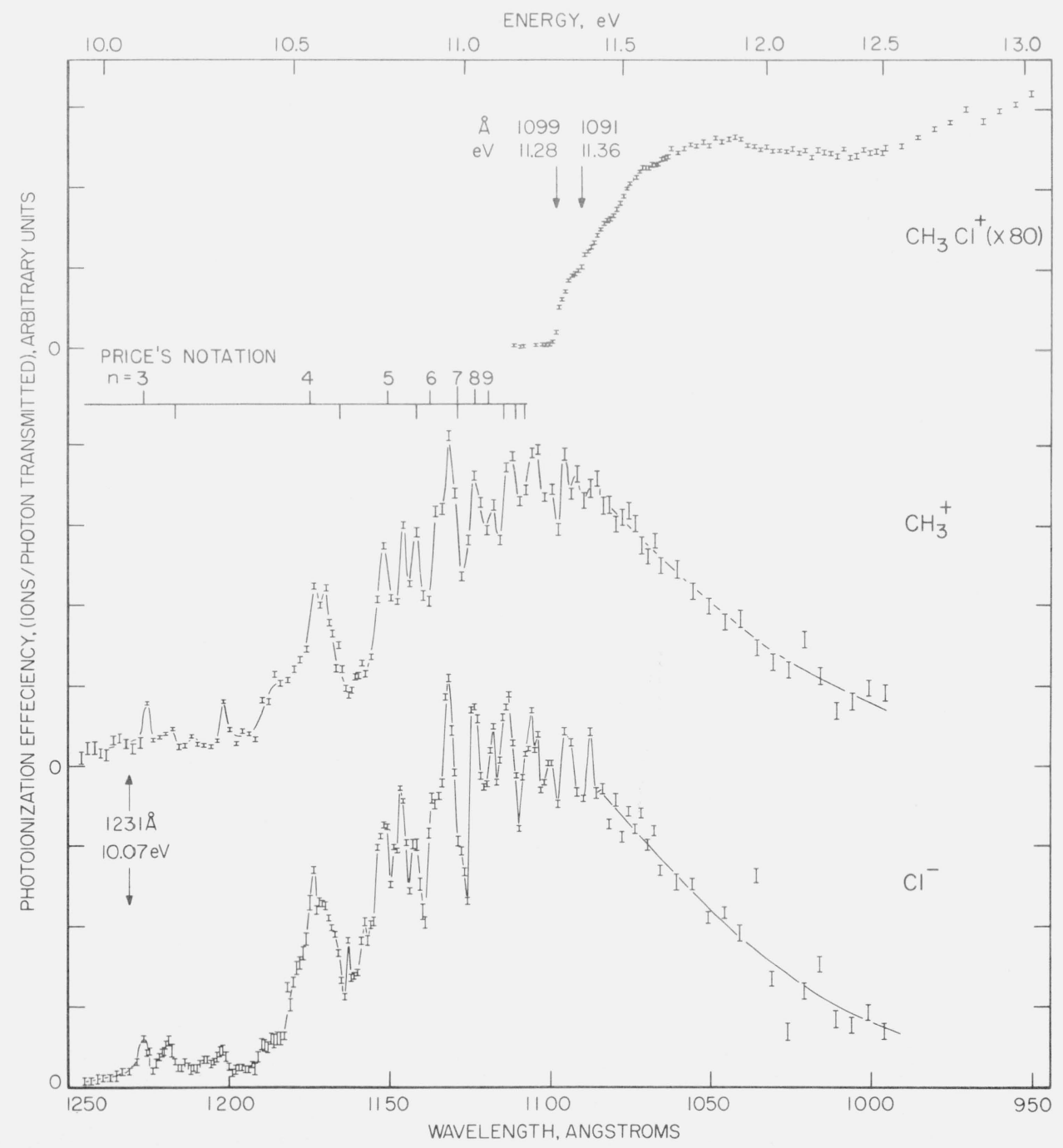

Figure 6. Photoionization yield curves for the methyl and halogen ions of methyl chloride formed by the ion-pair process. 


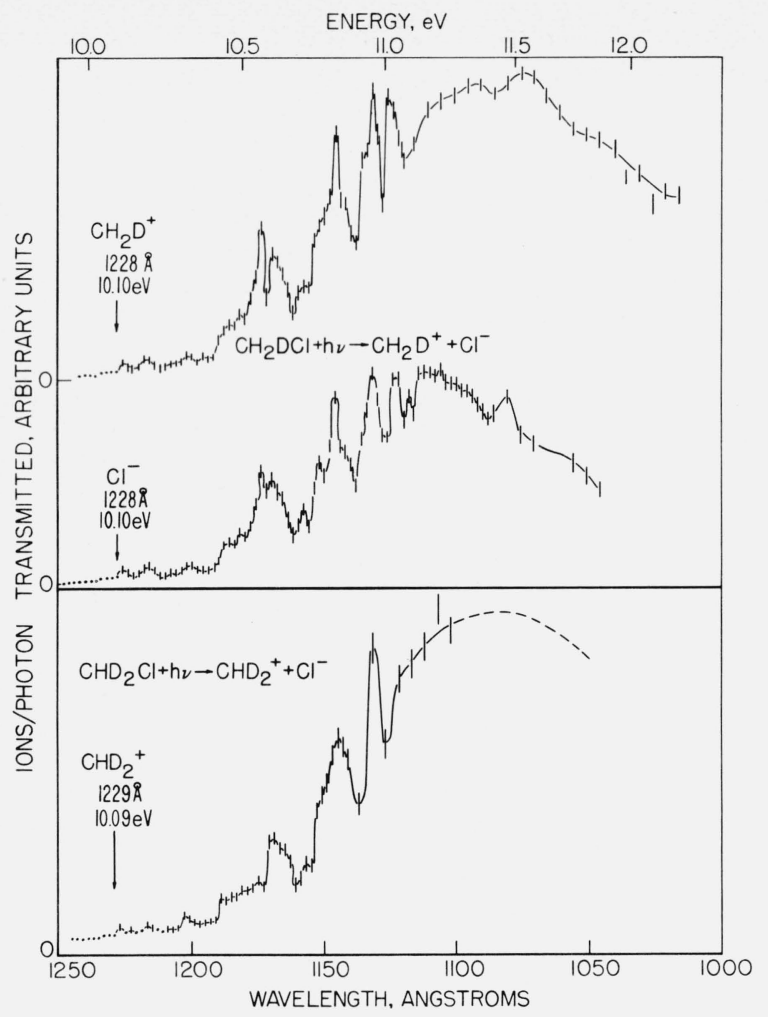

Figure 7. Photoionization yield curves for the methyl and halogen ions of $\mathrm{CH}_{2} \mathrm{DCl}$ and the methyl ion of $\mathrm{CHD}_{2} \mathrm{Cl}$ formed by the ionpair process.

TABLE 4. Comparison of ion-pair structure with $\mathrm{CH}_{3} \mathrm{Cl}$ Rydberg bands

\begin{tabular}{|c|c|c|}
\hline \multicolumn{2}{|c|}{ Ion-pair peaks } & Rydberg band energy $(\mathrm{eV})^{\mathrm{a}}$ \\
\hline $\begin{array}{l}\text { Angstroms } \\
1227 . \\
1219.5 \\
1201.5 \\
1185 . \\
1174 . \\
1170 . \\
1163.5 \\
1159.5 \\
1152.5 \\
1148 . \\
1143 . \\
1137 . \\
1132.5 \\
1124.5 \\
1119 . \\
1106.5 \\
1104.5 \\
1101.5 \\
1095 . \\
1088 .\end{array}$ & $\begin{array}{l}\mathrm{eV} \\
10.10 \\
10.17 \\
10.32 \\
10.46 \\
10.56 \\
10.60 \\
10.65 \\
10.69 \\
10.76 \\
10.80 \\
10.85 \\
10.90 \\
10.95 \\
11.02 \\
11.08 \\
11.20 \\
11.22 \\
11.25 \\
11.32 \\
11.39\end{array}$ & $\begin{array}{c}10.10 \\
10.18 \\
\\
\\
10.55 \\
10.63 \\
\\
\\
10.77 \\
10.86 \\
10.90 \\
10.98(2) \\
11.03 \\
11.07 \\
11.11 \\
11.15 \\
11.18 \\
\text { Limits: } \quad 11.22,11.30\end{array}$ \\
\hline
\end{tabular}

a Rydberg band energies are obtained from ref. 2a. They are compared with approximate peak values from figure 6 . The limits of the Rydberg series are from ref. $2 a$.

4. There is no isotope shift in the positions of the observed Rydberg interference so this indicates a lack of vibrational structure or at least that the vibrational progressions do not primarily involve the hydrogen atoms. Relative intensities of the Rydbergs do not vary much with isotopic substitution. It is pos- sible that the observed variation is due to line-width and noise problems.

The interpretation of these results can only be speculative as a calculation of the electronic structures of the excited states of methyl chloride is beyond our means. A very simplified diatomic model will be adopted and is schematically given in figure 8 . The only repulsive curve that is rapidly rising in the ionpair energy region is presumed to arise from the interaction of the unobserved $\mathrm{CH}_{3}\left({ }^{2} \mathrm{E}\right)$ state with the ground-state Cl. The Rydberg states to the $3 e_{1}^{-1}$ ion intersect this state and the configuration interaction is apparent up to the $3 e_{1}$ ionization limit. The Franck-Condon factor associated with the direct transition would be only slightly dependent on isotopic substitution. In order to provide for such an effect there is the final configuration interaction with the curve that connects asymptotically with the ion pair.

This model can be expressed more precisely in terms of the results of Mies [17]. His modification of the Fano continuum configuration theory includes the effect of direct inelastic coupling between the continua. The model we adopt for production of the ion-pair is dependent upon such coupling. As we noted above there are two continua to be considered, the ion-pair and the one leading to the $\mathrm{CH}_{3}\left({ }^{2} \mathrm{E}\right)$ molecule. There is an infinity of resonance states, the Rydberg states of $\mathrm{CH}_{3} \mathrm{Cl}$. Other continua involving fluorescence or dissociation are ignored. The transition amplitude for photoabsorption into the ion-pair channel is approximately given by

$$
t_{\alpha}^{-}=A_{\alpha, \beta}^{* *}\left(1-i \mathbf{K}_{\beta \beta}\right)^{-1}\left(t_{\beta}^{c}+\sum_{n} \frac{V_{n, \beta}}{E-E_{n}} t_{n}^{b}\right)
$$

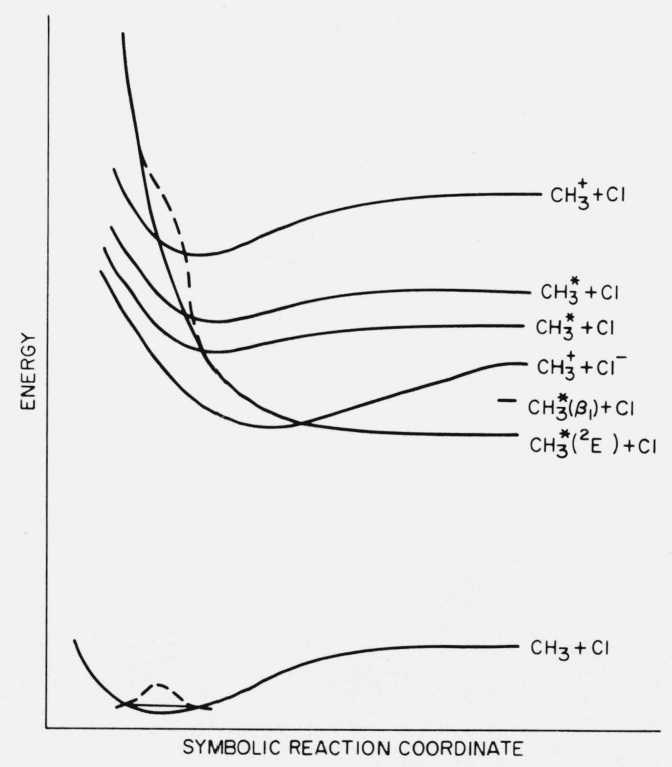

FIGURE 8. Schematic representation of the energy levels for a simplified model of $\mathrm{CH}_{3} \mathrm{Cl}$. 
The precise mathematical definition of these terms is given by Mies but we will try to explain their significance to the problem at hand. $\mathbf{K}$ is the reactance matrix and $(1-i \mathbf{K})^{-1}$ is a normalization term which conserves flux. The matrix element $V_{n, \beta}$ is a measure of the coupling of the continuum $\beta$ to the $n$th resonance state. The transition amplitudes $t_{\beta}$ and $t_{n}^{b}$ are the dipole matrix elements coupling the ground state to the $\mathrm{CH}_{3}\left({ }^{2} \mathrm{E}\right)_{\beta}$ continuum and the $n$th resonance state, respectively. $A_{\alpha, \beta}^{\circ}$ is the amplitude of inelastic scattering from the $\beta$ continuum to the $\alpha$ or ion-pair continuum.

It is our contention that there are no appreciable dipole couplings directly to the $\alpha$ continuum and in addition the resonance states do not couple to this continuum. Since the hydrogen vibrational modes are not excited there is no appreciable isotope effect in $t_{\beta}, t_{n}^{b}$ and, as required by the experimental results, in $V_{n, \beta}$. The fundamental resonance structure is not primarily related to the ion-pair state. As the $\beta$ continuum packet develops, there is a coupling to the $\alpha$ continuum given by $A_{\alpha, \beta}^{\circ}$. This amplitude is proportional to $V$, where $V$ is the matrix element which couples the two continua.

The radiationless transition converts translational into vibrational and electronic energy. In this model there is only a small isotope effect in the electronictranslational conversion. Under very simple assumptions $V^{2}$ is proportional to the Franck-Condon factor for the $\mathrm{N}-1$ vibrational degrees of freedom that are assumed not to participate in the transition. A large isotope effect is expected here. The transition occurs at large $\mathrm{CH}_{3}$ and $\mathrm{Cl}$ separations so that the subsequent couplings are neglected as the fragments go to infinity. The Franck-Condon factor of greatest importance is that for the pyramidal $\mathrm{CH}_{3}$ bending mode to the planar one since at the crossing it is assumed that almost free planar $\mathrm{CH}_{3}^{+}$is produced.

The Franck-Condon factor can be calculated for an oscillator that is primarily displaced. Siebrand [20] has provided numerical results for such a model. It can be shown that for a constant energy $E$ and excitation of a large number of quanta, the ratio of FranckCondon factors for the deuterium to hydrogen molecules is proportional to $\exp \left[-k\left(\frac{\mu_{I}}{\mu_{I I}}\right)^{1 / 2} E\right]$ where $\mu$ is the effective reduced mass for the normal frequency. The reduced masses can be replaced by the ratio of frequencies $\omega_{H} / \omega_{l}$. Depending on the number of quanta excited and the details of the frequencies, it would be possible to fit the observed isotope effect. Obviously the transition probability is less for the deuterated species and is smaller the more deuterium atoms are substituted. As the distribution of translation-vibrational energy is determined by details in the force constants and equilibrium positions of which we are ignorant, it can only be hoped that further experimentation would attempt to determine the relative kinetic energy. A knowledge of the distribution of kinetic energies for a given frequency, $\omega$, would go a long way toward establishing the validity of this model and make quantitative work meaningful.

This model predicts the production of the first excited $\mathrm{CH}_{3}\left({ }^{2} \mathrm{E}\right)$ state with the same resonance structure that is found for the ion-pair. The continuum shown by Price $[2 \mathrm{a}$ ] as starting at about $10 \mathrm{eV}$ can be attributed to this excited state. Transitions from the pyramidal excited state are not forbidden to the ground state and could be observed. In figure 8 we have assumed that the ${ }^{2} \mathrm{E}$ state is lower than all observed [21] Rydbergs of $\mathrm{CH}_{3}$. This may not be true but the ${ }^{2} \mathrm{E}$ asymptote should be less than that for the ion-pair. The relative position with respect to the Rydberg $\beta_{1}$ state would have an important bearing on the production of the $\beta_{1}$ state by a transition to the $\beta$ continuum.

\section{Thermodynamic Properties}

The simplest use of the data is to determine bond energies and ionization thresholds. The experimental data on the ionization thresholds are summarized in table 5. Where possible, calculated heats of formation of ions are given. In the two cases where accurate ionization thresholds have been obtained by analysis of a Rydberg series [2a] the values of the PE onset is in good agreement for the bromide and is about 0.1 $\mathrm{eV}$ greater in the case of the chloride.

When threshold values for fragment ions are used uncritically without concern for the distribution of energy in either internal modes or in relative kinetic energy, or for the transition probabilities to a given vibrational-rotational level, bond energies can be deduced. These are summarized in table 6 . The required atomic halogen ionization energies are obtained from Huffman's recent report [22] and the NBS compilation [23]. The ionization energy for $\mathrm{CH}_{3}, I\left(\mathrm{CH}_{3}\right)$ $=9.843 \mathrm{eV}\left(227.0 \mathrm{kcal} \mathrm{mol}^{-1}\right)$ is that due to Herzherg [21]. Heats of formation of neutral species are taken from "Selected Values of Chemical Thermodynamic Properties" [24]. Note that Chupka [19] has evidence that in some simple bond dissociations the rotational energy distribution contributes to the threshold ion production. On this basis bond energies would have to be raised about $0.02 \mathrm{eV}$. This is not done here because with present resolution such effects cannot be distinguished.

For $\mathrm{HCl}$, the threshold value of the $\mathrm{Cl}^{+}$ion is taken as the $\Delta H_{0}$ for the reaction shown in table 5 . Subtracting the ionization energy, $I(\mathrm{Cl})=12.967 \mathrm{eV}(299.0 \mathrm{kcal}$ mol $^{-1}$ ) gives $\Delta H f_{0}^{\circ}(\mathrm{Cl})=27.2 \mathrm{kcal} \mathrm{mol}^{-1}$. Thus, we calculate $D_{0}(\mathrm{H}-\mathrm{Cl})=100.8 \mathrm{kcal}^{-1}(4.37 \mathrm{eV})$. To the extent that this is an accurate value of the heat of formation, we may also obtain an improved value for $D_{0}(\mathrm{Cl}-\mathrm{Cl})=54.4 \mathrm{kcal} \mathrm{mol}^{-1}(2.36 \mathrm{eV})$. Our $D_{0}(\mathrm{H}-\mathrm{Cl})$ is $0.06 \mathrm{eV}$ less than the values quoted by Herzberg [25] and Cottrell [26]. The $D_{0}\left(\mathrm{H}-\mathrm{Cl}^{+}\right)$is greatly different from Herzberg's value but this is due to the inaccurate value of $I(\mathrm{HCl})$ used in the thermochemical cycle.

Unfortunately, the threshold values of the observed fragmentations of the methyl halides (possibly excepting $\mathrm{CH}_{3} \mathrm{Br}$ ) apparently do not yield direct bond dis- 
TABLE 5. Summary of threshold energies for hydrogen chloride and methyl halides

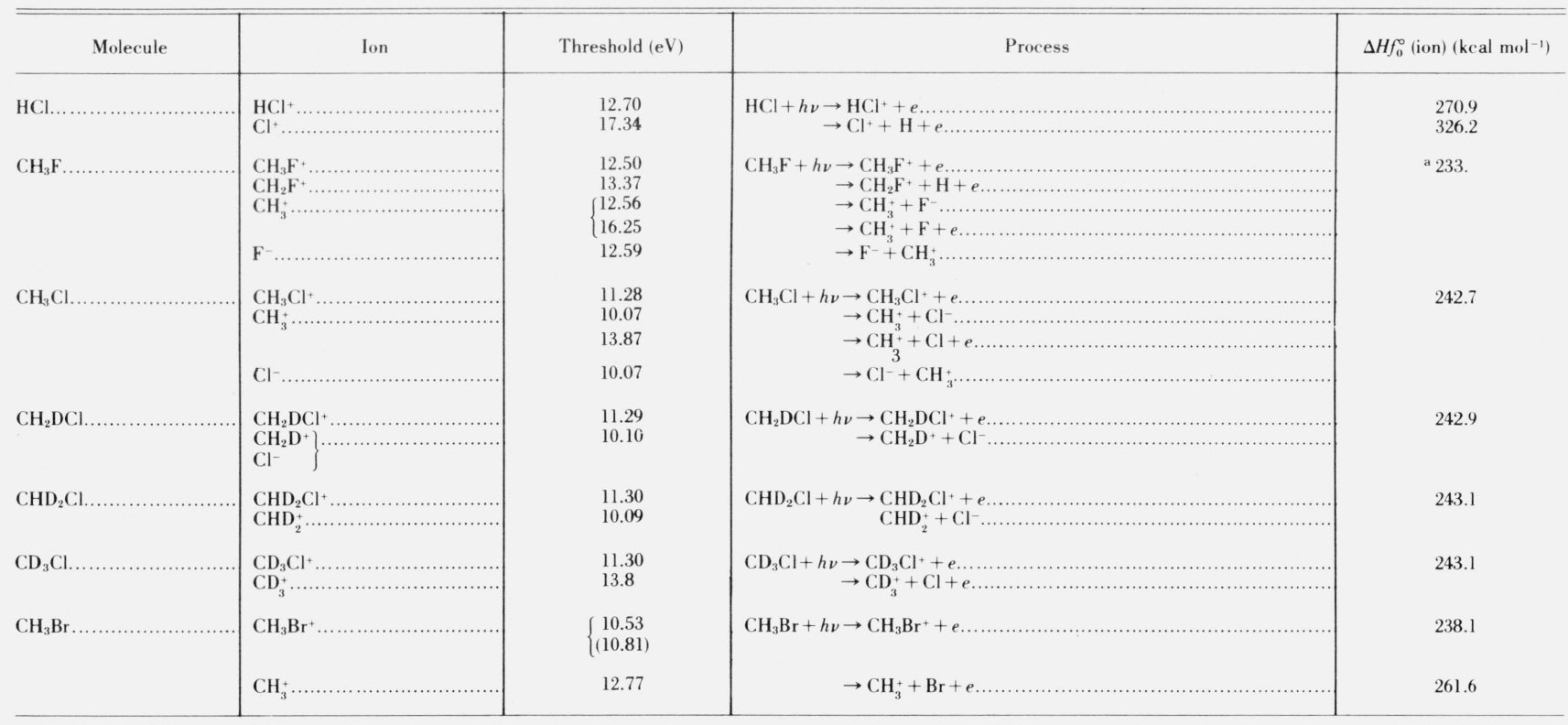

a Assuming $\Delta H f\left(\mathrm{CH}_{3} \mathrm{~F}\right)=-55 \mathrm{kcal} \mathrm{mol}^{-1}$ from ref. 27.

TABLE 6. Summary of observed and calculated bond dissociation energies

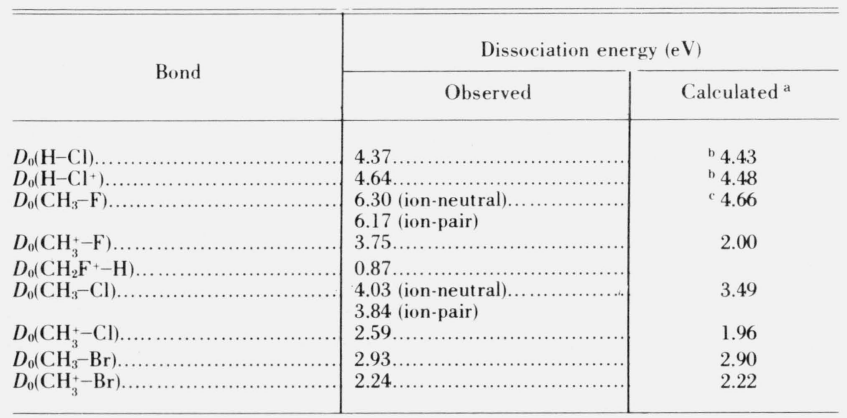

a Calculated from usual summation of heats of formation given in ref. 24.

${ }^{\mathrm{b}}$ Reported in ref. 25.

${ }^{c} \Delta H f_{0}^{\circ}\left(\mathrm{CH}_{3} \mathrm{~F}\right)=-55 \mathrm{kcal} \mathrm{mol}^{-1}$ from ref. 27 .

sociation energies. The heats of formation of the molecules, and of the related radicals and ions are generally established well within the uncertainties in the present measurements. A comparison of the observed $\mathrm{CH}_{3}-X$ bond dissociation energies summarized in the second column of table 6 with values calculated from heats of formation or obtained by spectroscopic means indicate that excess energy is included in most of the dissociative ionization processes. We had previously noted that the dissociation occurs from the excited ion state. The excess energy decreases from $1.7 \mathrm{eV}$ in $\mathrm{CH}_{3} \mathrm{~F}$ to about $0.5 \mathrm{eV}$ in $\mathrm{CH}_{3} \mathrm{Cl}$ and is probably insignificant in $\mathrm{CH}_{3} \mathrm{Br}$. The excess energy is observed in ion-pair as well as in ion-neutral processes. The appearance of kinetic energy with the formation of $\mathrm{CH}_{3}^{+}$ions in the fluoride and chloride compounds was reported in an earlier electron-impact study [28]. the magnitude decreased from fluoride to chloride and was immeasurable $(<0.2 \mathrm{eV})$ in the bromide and iodide. However, in the case of the fluoride, a lowerenergy threshold was also observed in both the ionneutral and ion-pair processes that resulted in a $\mathrm{H}_{3} \mathrm{C}-\mathrm{F}$ bond dissociation energy of $4.7 \mathrm{eV}$. This lower-energy threshold is not observed in the present study.

\section{Conclusion}

The behavior of the homologous series is disappointingly specific. The fluoride ion-pair production is from a resonance state which also branches into the ion-neutral process. Neutral predissociation probably dominates the resonances below ionization threshold as ion and Rydberg bond energies are very low. This is completely analogous to the methane case. In neither the fluoride nor the bromide cases, though, is the direct repulsive transition observed.

The significance of autoionization in these systems is undeniable. However, the data emphasize the need for caution in the interpretation of this autionization. If no observation of the ion-pair process had been made, the threshold would be considered a direct process and no thought of resonance states would arise. As it is, resonance neutral states would appear to contribute over the entire threshold region not only at the positions where peak-like structure is apparent.

Autoionization between the doublets also behaves in an unusual manner. Structure is apparent in the bromide which can be correlated with Rydberg levels reported by Price. However, the chloride gives no evidence of such autoionization. In the analogous argon curve at the same resolution, the doublet splitting is not observable. However, for the chloride there is a step at about the right energy. Perhaps, this 
is a vibrationally excited state superimposed on the doublet structure.

The thermochemical results more closely bound the ionization and bond energies although the role of rotational energy must still be considered. But as always, certain processes can be shown to occur from excited states and lead to excited states. This is the case for $\mathrm{D}\left(\mathrm{CH}_{3}-\mathrm{F}\right)$ and $\mathrm{D}\left(\mathrm{CH}_{3}-\mathrm{Cl}\right)$. To assist in interpreting such bond energy deductions or in determining inner ionization energies all ancillary information is required. In particular, approximate HartreeFock calculations of the electronic structure and inner ionization thresholds are very helpful especially as such calculations are essentially routine. From the data and the ionization threshold extremes, it is apparent that experimental determination of the inner onsets is not possible, at least at this resolution.

\section{References}

[1] Price, W. C.. Proc. Roy. Soc. (London) 167, 216 (1938).

[2] (a) Price, W. C., J. Chem. Phys. 4, 539 (1936); (b) Stokes, S., and Duncan, A. B. F., J. Amer. Chem. Soc. 80, 6177 (1958); (c) Kimura, K., and Nagakura, S., Spectrochimica Acta 17, 166 (1961).

[3] Dibeler, V. H., and Walker, J. A., J. Chem. Phys, 43, 1842 (1965).

[4] Morrison, J. D., Hurzeler, H., Inghram, M. G., and Stanton, H. E., J. Chem. Phys. 33, 821 (1960).

[5] Dibeler, V. H., and Reese, R. M., J. Res. NBS 68A, 409 (1964).
[6] Dibeler, V. H., and Walker, J. A., J. Opt. Soc. Am. 57, 1007 (1967).

[7] Mulliken, R. S., Revs. Mod. Phys. 4, 6 (1932).

[8] Cade, P. E., and Huo, W. M., J. Chem. Phys. 47, 649 (1967).

[9] Norling, F., Z. Physik 104, 638 (1937).

[10] Norling, F., Z. Physik 106, 177 (1937).

[11] Morrison, J. D., J. Chem. Phys. 19, 1305 (1951).

[12] Fox, R. E., J. Chem. Phys. 32, 385 (1960).

[13] Halmann, M., and Laulicht, I., J. Chem. Phys. 43, 1503 (1965).

[14] Nesbet, R. K., J. Chem. Phys. 41, 100 (1964).

[15] Fano, U., Phys. Rev. 124, 1866 (1961).

[16] Fano, U., and Prats, F., J. Natl. Acad. Sci. India A33, 553 (1963).

[17] Mies, F., private communication.

[18] Krauss, M., International Mass Spectrometry Conference, Berlin (1967). The eigen functions are available upon reasonable request.

[19] Chupka, W. A., J. Chem. Phys. 48, 2337 (1968).

[20] Siebrand, W., J. Chem. Phys. 46, 440 (1967).

[21] Herzberg, G., Proc. Roy. Soc. (London) A262, 291 (1961).

[22] Huffman, R. E., Larrabee, J. C., and Tanaka, Y., J. Chem. Phys. 47, 856 (1967).

[23] Moore, C. E., NBS Circ. 467 (1952).

[24] Wagman, D. D., Evans, W. H., Parker, V. B., Halow, I., Bailey, S. M., and Schumm, R. H., NBS Tech. Note 270-3 (1968).

[25] Herzberg, G., Molecular Spectra and Molecular Structure I. Spectra of Diatomic Molecules (D. Van Nostrand Co., Inc., New York, 1950).

[26] Cottrell, T. L., The Strengths of Chemical Bonds (Academic Press Inc., New York, 1958).

[27] Benson, S. W., J. Chem. Educ. 42, 502 (1965).

[28] Dibeler, V. H., and Reese, R. M., J. Res. NBS 54, 127 (1955) RP2573.

(Paper 72A4-502) 